\title{
Imaging of steep flanks by focal sources
}

Jan Thorbecke*, Delft University of Technology, Department of Applied Sciences, Section of Acoustical Imaging and Sound Control, The Netherlands

\section{Summary:}

One-way recursive wavefield extrapolation and migration has many advantages over other wavefield extrapolation methods. The method can handle complex models and is robust. However, the extrapolation of turning waves is one important omission of one-way wavefield extrapolation In this paper a new hybrid approach for the imaging of steep salt flanks is presented: CFP redatuming is used to redatum the data to a vertical array, and one-way wavefield extrapolation, using a rotated velocity field, is used to image the steep flank. A simple example is given for a synthetic data-set calculated for a model which includes a steep flank.

\section{Introduction:}

Virtual sources can be simulated in the subsurface using a source array at the surface (Berkhout, 1992; Rietveld et al., 1992). These virtual sources can be focussed (combined) again to illuminate specific parts of the subsurface. In the last section of Chapter 5 of Thorbecke (1997) an idea to image steep flanks was illustrated in a figure (repeated here in Figure 1), but has never been worked out. The last year this idea has been worked out in a slightly different way. The idea from

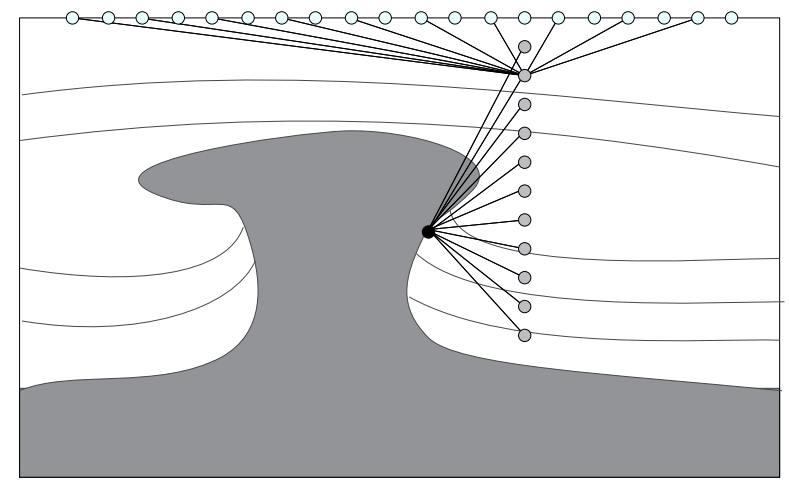

Figure 1: Focusing of CFP gathers to illuminate the flank of a salt dome. If direct reflection information of the flank of the salt dome is measured at the surface then it is possible to unveil this information by a secondary focusing step. (from Chapter 5 of Thorbecke, 1997)

1997 was: "By choosing several focus points close to each other, for example on the same lateral position but at different depth levels, and making use of the interpretation that a CFP gather for focusing in emission is the response of a source at the focus point and receivers at the surface, a second focusing step can be applied on the CFP gathers. In Figure $\square$ an application of this idea is sketched; close to a flank of a salt dome focus points are chosen at several depth levels (the grey dots). After the calculation of the CFP gathers for focusing in emission a second focusing step is used to focus the focus point responses on a point positioned on the flank of the salt dome (the black dot). Application of this idea can be useful if the macro model is already accurate and one wants to image the flank of the reflector better. Note that in this method only the direct reflections of the flank are used and not the second order reflections.

Krebs et al. (1996) have shown that the use of accurate migration operators can improve the quality of the image of the flank of a salt dome. In their paper accurate migration operators are obtained by measuring the focus point responses, from a well close to the flank of the salt dome, at the surface. In the CFP method accurate operators can be obtained by updating the focusing operator."

In short the idea boils down to the fact that an (vertical) array of focal points (FP's), with corresponding CFP-gathers and CFPoperators, is used to redatum surface data to the array of FP's. The response of this array is used to illuminate steeps flanks. The CFP redatuming, followed by imaging is worked out in this abstract and will be illustrated by the imaging of a steep flank in a synthetic data set.

\section{Target focusing; focusing of CFP gathers}

To redatum a data set recorded at the surface to a vertical array, focusing operators are needed which contain the propagation effects between the vertical array, and the acquisition positions at the surface. A first focusing step creates CFP gathers which have virtual source positions on the vertical array and real detectors on the surface. After this first step a second focusing step is used, using the same focusing operators, but now with CFP gathers as input. After this second focusing step the focal point acts as a source position (where the focussing operator is confocal $(n=m)$ with the focal point) and the other points in the vertical array, are receiver locations (where the focusing operators are bifocal $(n \neq m)$ with the focal point). The CFP gather and the redatumed CFP gather are represented by

$$
\begin{aligned}
& \mathbf{P}_{j}\left(z_{0}, z_{n}\right)=\mathbf{P}\left(z_{0}, z_{0}\right) \mathbf{F}_{j}\left(z_{0}, z_{n}\right), \\
& \mathbf{P}_{j}\left(z_{m}, z_{n}\right)=\mathbf{F}\left(z_{m}, z_{0}\right) \mathbf{P}_{j}\left(z_{0}, z_{n}\right)
\end{aligned}
$$

respectively. The constructed gathers $\mathbf{P}_{j}\left(z_{n}, z_{m}\right)$ can be migrated using a standard one-way shot record migration program, where the velocity model is rotated to become aligned with the new positions of the vertical array.

Zhang and McMechan (1997) used a similar approach, and also used a rotated velocity model of $90^{\circ}$ to extrapolate the energy associated with turning waves, but they did not use a (CFP) redatuming step as introduced in this chapter. The redatuming step has two advantages:

- a smaller aperture is needed to migrate the reflection energy from the steep flank,

- the energy after redatuming is better focussed, which 
Imaging of steep flanks by focal sources

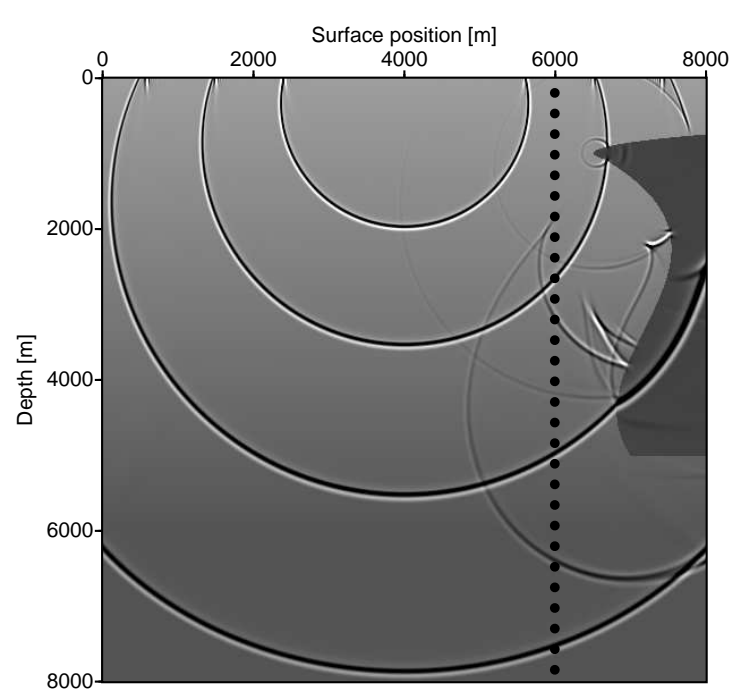

Figure 2: Salt dome model embedded in a medium with a vertical velocity gradient. The vertical array at $x=6000$ shows the 533 locations $(\Delta z=15)$ of the FP's, each FP being connected to a CFP-gather. Note the complex, multi-valued, wavefront from the reflection of the steep salt flank in the snapshots.

makes the (least squares) imaging condition better defined and more stable.

\section{Example of a flank model}

To illustrate the CFP approach a synthetic steep flank model, shown in Figure 2 is used. In this model 534 shots were modeled (using an acoustic finite-difference program) with a fixedspread acquisition, ranging from 0 to $8000 \mathrm{~m}$, and a source and receiver spacing of $15 \mathrm{~m}$. The model consists of a background model with a vertical velocity gradient and a salt-like structure on the right hand-side of the model. The recorded wavefield from shots on the left hand-side of the model, will contain energy from reflected turning waves. The dotted line in Figure 2 indicates the vertical positions of the focal points (FP's), each FP being connected to a CFP-gather. The vertical distance between the FP positions in the vertical array is chosen $(\Delta z=15$ m.). These vertical FP positions will become the 'new' acquisition plane. The snapshots in Figure 2 show that the reflection of the steep flank contains multiple arrivals. Figure 3 shows a shot record with a source position in the outer left part of the model $(x=105 \mathrm{~m})$. The reflection from the top of the salt and multiple arrivals from the steep flank are clearly visible.

The focusing operators, used to construct the CFP gathers at the vertical array, are calculated using a ray-based modeling program. These calculated focussing operators will correctly follow the turning waves through the model. The velocity model used for the ray-based modeling only contains the background model: a depth velocity gradient of $0.4 \mathrm{~m} / \mathrm{s}$ per meter ranging from 0 to 6000 meter and starting at $z=0$ with $1500 \mathrm{~m} / \mathrm{s}$. Usually the velocity gradient along the side(s) of a salt structure is well known. On positions of the vertical array where the focal point coincides with a reflecting boundary,

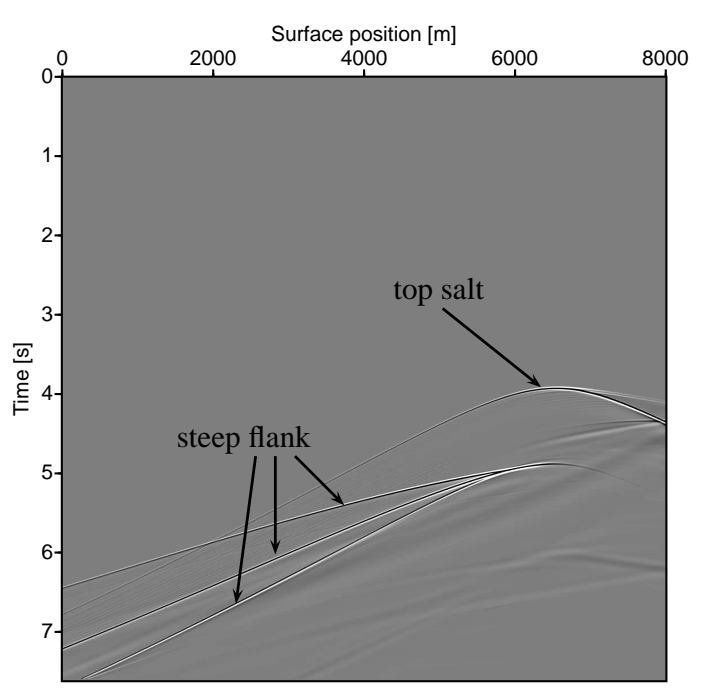

a) Shot record source at $x=105, z=0$.

Figure 3: Shot record from the far left side of the model. Multiple arrivals in the turning wave event occur around $6 \mathrm{~s}$.

the calculated operator can be verified using the CFP principle of equal traveltime. If one has measurements from buried detectors, then the first arriving event in these measurements (Xiao et al.,2006) can also be used to constrain and verify the calculated focussing operators.

The CFP gather in Figure 4 $\mathrm{l}$, includes reflection energy from the steep flank which has propagated through the vertical gradient medium. The reflection event in the CFP gather around $4 \mathrm{~s}$. originates from the steep flank of the salt. After constructing all CFP gathers at the defined vertical array, the redatuming step is completed by using the focusing operators a second time. Applying this redatuming step to all CFP gathers gives a new pre-stack volume. For one redatumed position the constructed shot record is shown in Figure $4 \mathrm{~b}$. This redatumed CFP gather can be interpreted as the response of a source at $x=6000, z=1500$ with receivers at the vertical line $x=6000, z=[15-7995]$. In this redatumed shot record multi-valued arrivals from the synclinal structure of the flank are visible and indicated with an arrow.

The application of this redatuming process gives 533 redatumed CFP gathers, each with 533 receivers. To verify the velocity model (or focussing operators) of the salt flank structure the redatumed data set can also be used to construct CFP gathers with FP's on the flank, and check 'the principle of equal traveltime'. Indicated in Figure 5 a focal point (blue star) on the flank of the salt dome is chosen at $z=3000, x=7330$, a focusing operator is calculated, and CFP gather constructed. Figure 6 shows the CFP gather with its focussing operator overlaid. Note that the operator verification, using the reflections from the steep flank, can lead to accurate focusing operators.

Migrating the redatumed CFP gathers with a pre-stack depth migration algorithm gives the image shown in Figure $7 \mathrm{~b}$. For comparison in Figure 7t the same area for the pre-stack depth migration result is shown for surface shot record migration 


\section{Imaging of steep flanks by focal sources}
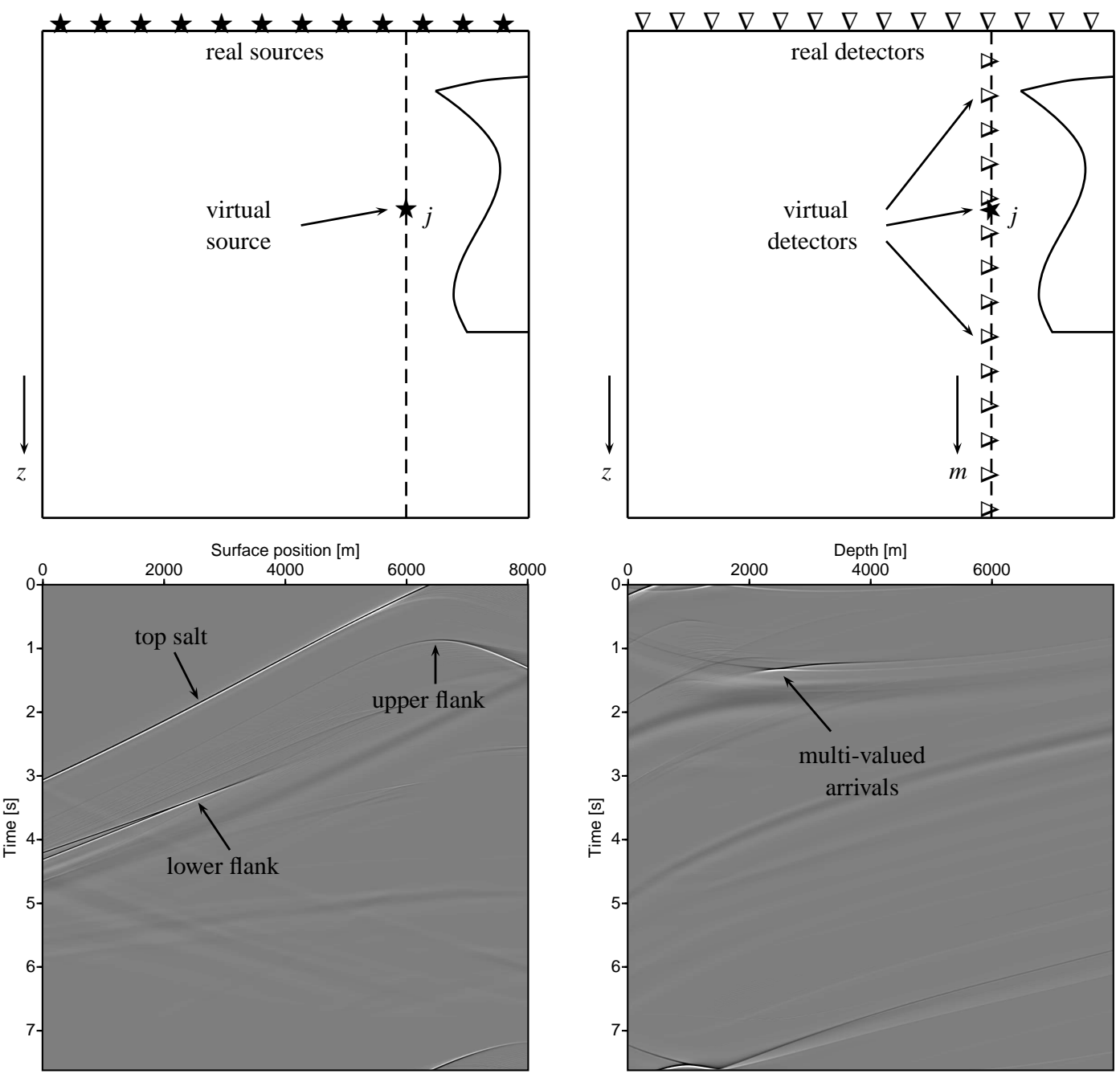

a) CFP gather with FP at $(6000,1500)$

b) Redatumed CFP gather

Figure 4: CFP gather for focal points at depth of $1500 \mathrm{~m}$ after the first (a) focussing step and after redatuming to the vertical array at $x=6000$ (b). Note that the horizontal axis in a) represents surface position of the real sources, and in b) the horizontal axis represents depth positions of the virtual sources.

(using the same one-way wavefield extrapolation operators (Thorbecke et al., 2004)). Note the better imaging of the steep flank of the salt structure. One-way extrapolation operators, applied on recorded data at the surface and using a regular grid, cannot handle turning waves, so the energy of the turning waves, which are visible in Figure 3 is suppressed by the one-way operators, and not imaged in Figure 7 . Note that reflections of the top and bottom of the salt, which has travelled through the salt, are nicely imaged. Note also that parts of the steep flank are imaged due to the presence of diffraction energy originating from course blocks in the finite difference model. This imaged diffraction energy is visible as horizontally oriented events which are not aligned with the steep flank.

\section{Conclusion}

Redatumed CFP gathers can be used to image complex geological structures which contain steep flanks. The proposed procedure consists of three steps: First a slant or vertical or curved array of focal points with their CFP gathers is constructed. Second the CFP gathers are redatumed to the array positions. The redatumed CFP gathers represent a fixed acquisition spread along the array of focal points. Thirdly, these redatumed CFP gathers are input to standard velocity analysis and then migrated by a standard shot record migration program. Note that the position of the structure can be verified by using the principle of equal travel time. The obtained migrated image will show information of high angles and overturned waves when these are present in the data. For an optimal imaging and detailed velocity analysis the virtual array can be designed for any specific geological structure.

\section{Acknowledgements}

The author would like to thank the sponsors of the DELPHI consortium for their financial support. 

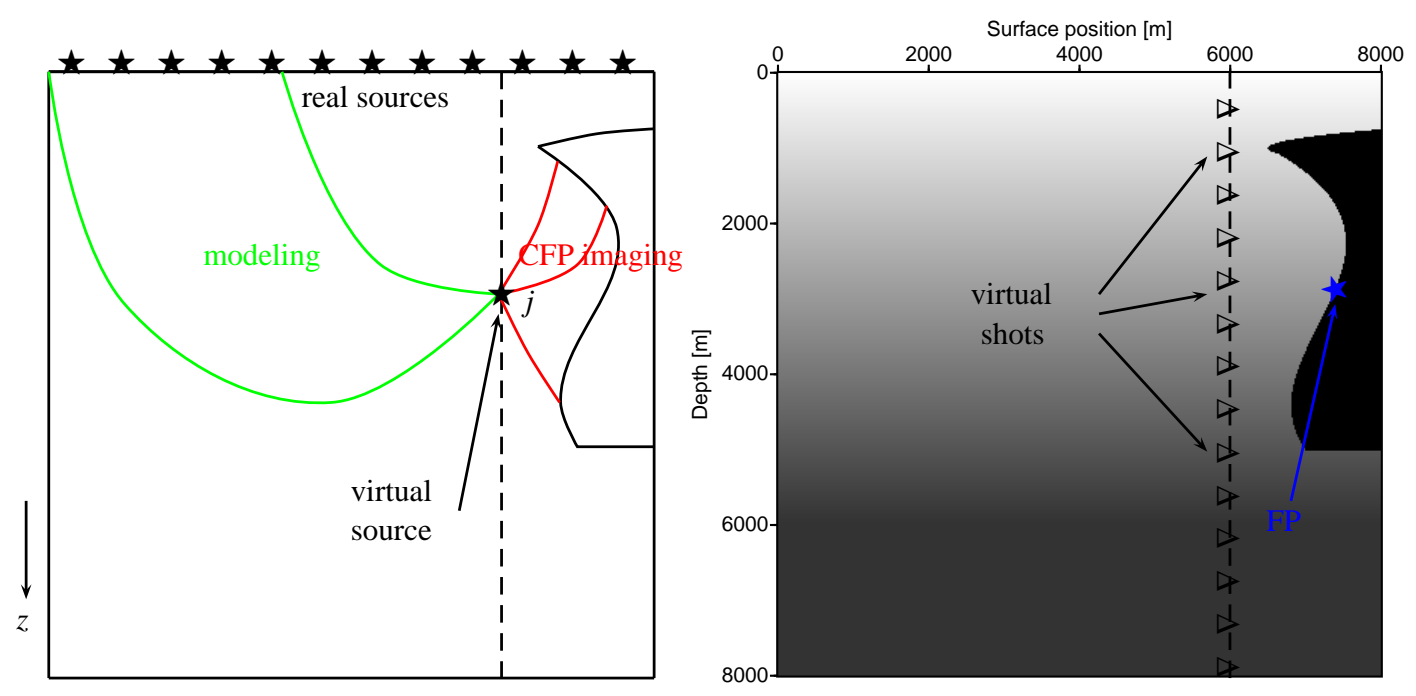

Figure 5: For the imaging of steep flanks a two step procedure is proposed. In the first step focusing operators (one-way Green's functions) are modeled in a background model and surface data is redatumed to a vertical array of focal points. The second step standard (CFP) migration analysis is used to image the steep reflector. To check the velocity model, used to migrate the redatumed data, CFP analysis can be used for focal points (blue star) on the flank.

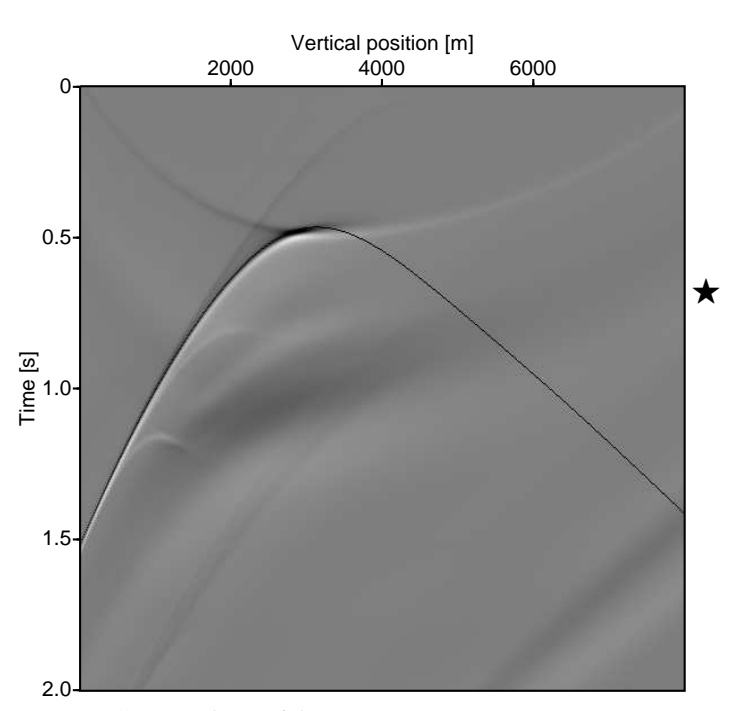

a) CFP gather with FP at $(7330,3000)$.

Figure 6: Focusing analysis on the rotated flank of the salt structure can be used to check the correctness of the focusing operators. The operator (shown as the black line superimposed on the CFP gather) and the CFP gather have the same traveltime indicating that the correct operator was used.
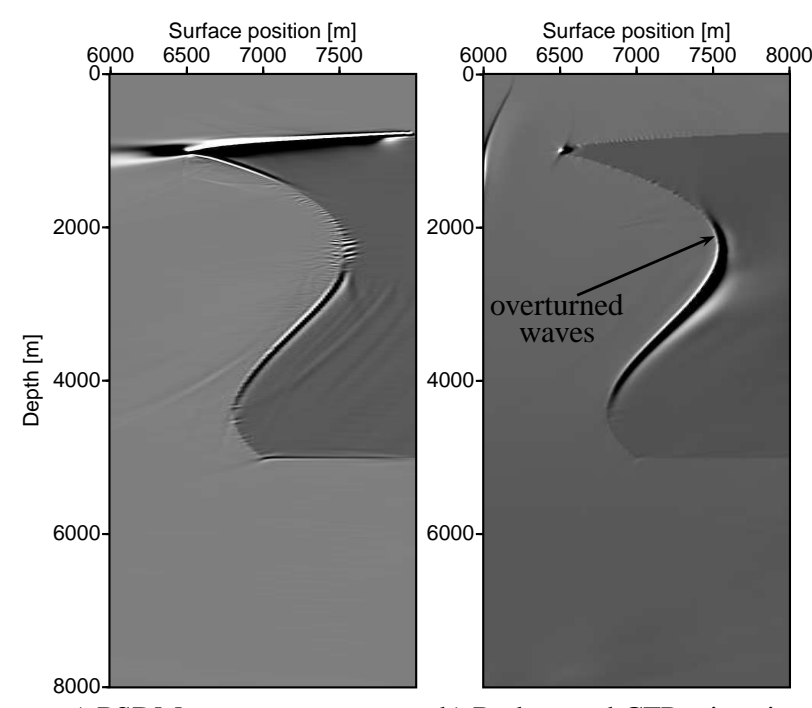

a) PSDM

b) Redatumed CFP migration

Figure 7: Comparison between conventional shot record (a) and redatumed CFP gather (b) migration. Note that the CFP gather images the flank much better and also images the turning waves (indicated with an arrow). Due to the diffractors at the grid corners (staircase effect) of the coarse grid in the finite difference model, the steep flank in (a) is partly imaged due to the presence of diffraction energy. This diffraction energy is also clearly present at the base of the structure. 


\section{Imaging of steep flanks by focal sources}

\section{REFERENCES}

Berkhout, A. J., 1992, Areal shot record technology: Journal of Seismic Exploration, 1, 251-264.

Krebs, J. R., D. R. Fara, and A. E. Berlin, 1996, Accurate migration using offset-checkshot surveys: 58th Ann. Internat. Mtg., Expanded Abstracts, Session:X020, Eur. Assoc. Expl. Geophys.

Rietveld, W. E. A., A. J. Berkhout, and C. P. A. Wapenaar, 1992, Optimum seismic illumination of hydrocarbon reservoirs: Geophysics, 57, 1334-1345.

Thorbecke, J., K. Wapenaar, and G. Swinnen, 2004, Design of one-way wavefield extrapolation operators, using smooth functions in WLSQ optimization: Geophysics, 69, 1037-1045.

Thorbecke, J. W., 1997, Common focus point technology: PhD thesis, Delft University of Technology.

Xiao, X., M. Zhou, and G. T. Schuster, 2006, Salt-flank delineation by interferometric imaging of transmitted P- to S-waves: Geophysics, 71, SI197-SI207.

Zhang, J. and G. A. McMechan, 1997, Turning wave migration by horizontal extrapolation: Geophysics, 62, 291-297. 


\section{EDITED REFERENCES}

Note: This reference list is a copy-edited version of the reference list submitted by the author. Reference lists for the 2008 SEG Technical Program Expanded Abstracts have been copy edited so that references provided with the online metadata for each paper will achieve a high degree of linking to cited sources that appear on the Web.

\section{REFERENCES}

Berkhout, A. J., 1992, Areal shot record technology: Journal of Seismic Exploration, 1, 251-264.

Krebs, J. R., D. R. Fara, and A. E. Berlin, 1996, Accurate migration using offset-checkshot surveys: 58th Annual International Conference and Exhibition, EAGE, Extended Abstracts, X020.

Rietveld, W. E. A., A. J. Berkhout, and C. P. A. Wapenaar, 1992, Optimum seismic illumination of hydrocarbon reservoirs: Geophysics, 57, 1334-1345.

Thorbecke, J., K. Wapenaar, and G. Swinnen, 2004, Design of one-way wavefield extrapolation operators, using smooth functions in WLSQ optimization: Geophysics, 69, 1037-1045.

Thorbecke, J. W., 1997, Common focus point technology: Ph.D. thesis, Delft University of Technology.

Xiao, X., M. Zhou, and G. T. Schuster, 2006, Salt-flank delineation by interferometric imaging of transmitted P- to Swaves: Geophysics, 71, SI197-SI207.

Zhang, J., and G. A. McMechan, 1997, Turning wave migration by horizontal extrapolation: Geophysics, 62, $291-297$. 